\title{
OCEANS' three: the ovarian job
}

$\mathrm{T}$ he lack of specific symptoms and early dissemination to the peritoneal cavity have made ovarian cancer the most lethal of all gynecologic malignancies. Even with cytoreductive surgery and platinumbased chemotherapy, a high percentage of patients relapse. Those patients who relapse after 6 months of receiving platinum-based chemotherapy are still considered to be sensitive to this treatment and re-treating them with the platinumbased regimen in combination with gemcitabine is current standard of care.

\section{The significant}

\section{improvement in PFS is of the highest magnitude seen to date in this disease 77}

Although the response rates to antiangiogenic agents in many cancer types have not been as successful as expected, ovarian cancer seems to respond well to antiangiogenic therapy. So well, in fact, that two phase III trials (GOG-0218 and ICON7) published in December of 2011 led to the approval by the EMA of bevacizumab-a monoclonal antibody targeting VEGF-as first-line treatment in combination with carboplatin and paclitaxel. Does this mean that bevacizumab, received in combination with the standard regimen of gemcitabine and carboplatin, could also be the way forward in the treatment of recurrent ovarian cancer (ROC)?

Carol Aghajanian has now reported a study that could well address this question. Encouraged by the results of previous phase II trials that had shown efficacy of bevacizumab in ROC, Aghajanian and her collaborators initiated the OCEANS (ovarian cancer study comparing efficacy and safety of chemotherapy and antiangiogenic therapy in platinum-sensitive recurrent disease) study, a randomized, double-blind, phase III trial aimed to further assess the efficacy of adding bevacizumab to the standard combination of carboplatin and gemcitabine. As the previous studies had reported a high percentage of gastrointestinal toxicity in the bevacizumab arms, the purpose of OCEANS was also to obtain and review extensive safety data. To this end, it was originally initiated as a phase II trial in which 484 women with ROC-and disease progression after 6 months of treatment with platinum-based chemotherapy - were randomly assigned to receive gemcitabine and carboplatin in combination with placebo $(n=238)$ or in combination with bevacizumab $(n=241)$. The patients received $6-10$ cycles of gemcitabine and carboplatin. Bevacizumab or placebo was started with the first cycle of chemotherapy and continued following completion of chemotherapy until the time of progression of disease or intolerable side effects.

After a 2-year follow up, the results revealed that adding bevacizumab to the standard combination of gemcitabine and carboplatin translates into a difference of 4 months in progression-free survival (PFS; 8.4 months and 12.4 months in the bevacizumab and placebo arms, respectively). Importantly, there was also a significant difference in overall response rate, with a response rate of $78 \%$ in the bevacizumab arm compared with only $57 \%$ in the placebo arm. "The significant improvement in PFS is of the highest magnitude seen to date in this disease", affirms Aghajanian.

This significant improvements in PFS of 4 months and response rate of more than $20 \%$ with the addition of bevacizumab, came at a cost of some serious adverse events, such as hypertension and proteinuria. In general, more patients in the bevacizumab arm (89\%) experienced grade $3-5$ adverse events than in the placebo arm (82\%), although there was no gastrointestinal perforation reported during treatment with bevacizumab.

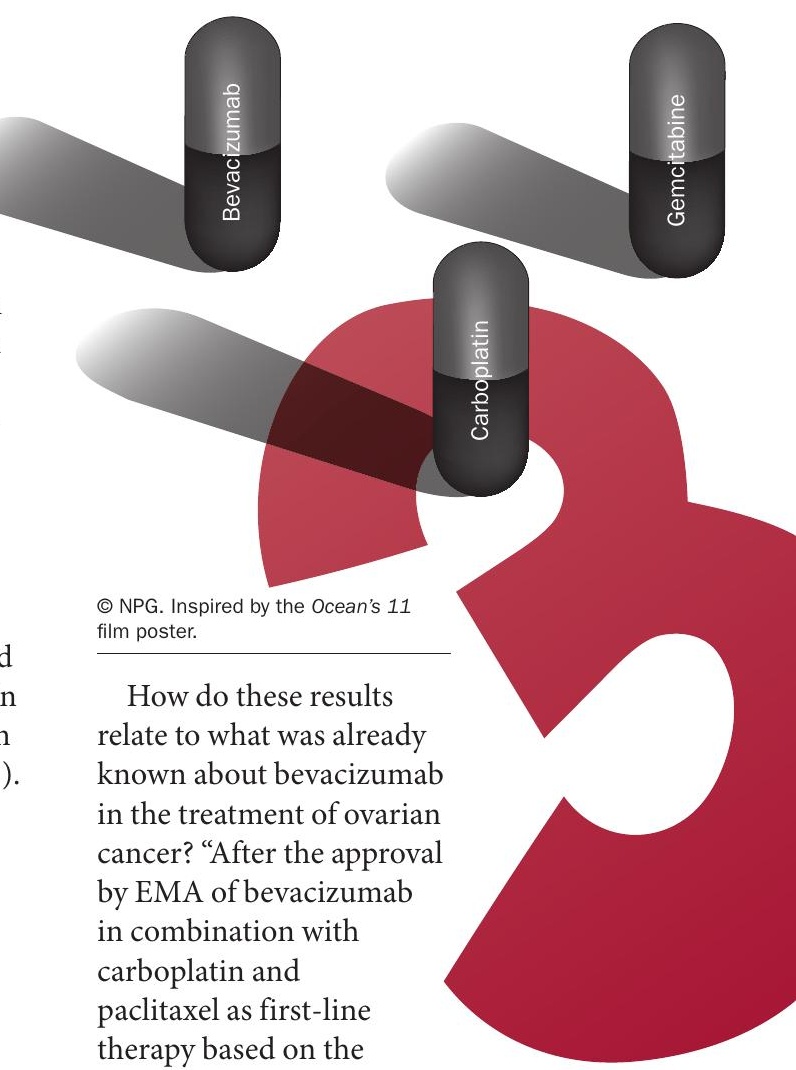

GOG-0218 and ICON7

positive phase III trials"-comments

Susana Banerjee, at the Royal Marsden in

London, UK- "this study demonstrates that in addition to the first-line setting, bevacizumab has clinically meaningful activity in platinum-sensitive recurrent ovarian cancer". She continues,

"a reasonable proposal for patients optimally debulked, and thus at a lower risk of early relapse, would be to reserve bevacizumab until first recurrence."

We now eagerly await the results of the AURELIA study, which is assessing the role of bevacizumab in platinum-resistant ovarian cancer and will be reported soon.

\section{Teresa Villanueva}

Original article Aghajanian, C. et al. OCEANS: a
randomized, double-blind, placebo-controlled phase III
trial of chemotherapy with or without bevacizumab in
patients with platinum-sensitive recurrent epithelial ovarian,
primary peritoneal, or fallopian tube cancer. J. Clin. Oncol.
doi:10.1200/JC0.2012.42.0505
Further reading Banerjee, S. \& Kaye, S. B. First-line
bevacizumab for ovarian cancer-new standard of care?.
Nat. Rev. Clin. Oncol. 9, 194-196 (2012)

